\title{
Theta series for indefinite quadratic forms over real number fields
}

\author{
by \\ Jeffrey Stopple (Santa Barbara, Cal.)
}

\begin{abstract}
0. Introduction. We wish to construct a theta function in an explicit way for a general quadratic form $q$ and lattice $\mathfrak{L}$ over a real algebraic number field. Two difficulties present themselves. The first is that if the class number of the ring of integers is not 1 , then the lattice $\mathfrak{L}$ need not be free. However, the underlying space is also a vector space over $\mathbb{Q}$, and $\mathfrak{L}$ is free as a $\mathbb{Z}$-module. This process of going down to $\mathbb{Q}$ is called the Scharlau transfer $\operatorname{tr}(q)$. We follow Eichler [2] in this. The second problem is that if the form $q$ is not totally positive, the usual sum over the lattice will not converge. This can be fixed by introducing the analogue of a spherical harmonic for the theta function to insure convergence. The complex variable $\tau$ must be split up into real and imaginary parts, so that the theta function is not necessarily holomorphic. The inversion formula is classically proved for $\operatorname{Re}(\tau)=0$ by Poisson summation and then analytically continued; this requires modifications in the nonholomorphic case. In this we imitate the paper by Vignéras [7], which solves the problem over $\mathbb{Q}$ in a very elegant way by studying the properties of Hermite polynomials. Vignéras notes the resulting theta functions are the same as the ones Weil constructed; the differential equations involved give a condition of the behavior of a certain function under the Weil representation. (In Schempp [4] a canonical orthonormal basis of the representation space for the Schrödinger representation of the Heisenberg group is given in terms of Hermite polynomials.)

As an application we consider in Section 5 base change lifting of automorphic forms to a real quadratic extension of a totally real number field, with the point of view of making all computations as explicit as possible.

The author would like to thank Lynne Walling for many helpful conversations. Part of this work was done with the support of a University of California, Santa Barbara Regents Junior Faculty Fellowship, and part was done while a guest of the Max Planck Institut für Mathematik, Bonn.
\end{abstract}


1. Notations. Since we are going to imitate Eichler's proof, it makes sense to follow his notation as closely as possible. We denote by

- $K$ a totally real number field, $[K: \mathbb{Q}]=n$. The conjugates of an element $\alpha$ of $K$ will be denoted $\alpha^{j}, j=1, \ldots, n$,

- $\mathcal{O}$ the ring of integers with class number $h$,

- $V$ a vector space over $K$ of even dimension $2 k$ with typical element $\mathfrak{v}$,

- $\mathfrak{L}$ a lattice of full rank $2 k$,

- $q$ a quadratic form on $V$ with coefficients in $K$. For notational convenience we assume the form $q$ has the same signature $(s, t)$ at each embedding of $q$ into $\mathbb{R}$,

- $\langle\mathfrak{v}, \mathfrak{w}\rangle$ the corresponding bilinear form such that $q(\mathfrak{v})=\langle\mathfrak{v}, \mathfrak{v}\rangle$,

- $\mathfrak{b}_{\mu}$ for $\mu=1, \ldots, 2 k$ a basis of $V$ over $K$,

- $\gamma_{\nu}$ for $\nu=1, \ldots, n$ a basis of $K$ over $\mathbb{Q}$,

- $\mathfrak{l}_{1}, \ldots, \mathfrak{l}_{d}$ a basis for $\mathfrak{L}$ as a $\mathbb{Z}$-module, where $d=2 k n$,

- $\Phi=\operatorname{det}\left(\left\langle\mathfrak{l}_{\mu}, \mathfrak{l}_{\nu}\right\rangle\right)$,

- $\tau=\left\{\tau^{i}\right\}$ an element of the upper half plane $\mathbf{H}^{n}$. If we write $\tau=u+i v$ it should be understood that $u$ and $v$ are the corresponding vectors of real and imaginary parts.

The transpose of a matrix $A$ is denoted by ${ }^{t} A$, and $A[x]=x A^{t} x$. We let $F$ be the symmetric matrix $\left\{q\left(\mathfrak{b}_{\mu}, \mathfrak{b}_{\nu}\right)\right\}$ so that for $\mathfrak{v}=\sum \xi_{i} \mathfrak{b}_{i}$ in $V$ corresponding to the row vector $\xi$ in $K^{2 k}$ we have $q(\mathfrak{v})=F[\xi]$.

We let $G$ be a $d \times d$ matrix of $2 k \times 2 k$ blocks, where the block in row $i$ and column $j$ is the scalar matrix $\gamma_{i}^{j} I_{2 k}, i, j=1, \ldots, n$. Decomposing the vector $\mathfrak{v}=\sum \xi_{i} \mathfrak{b}_{i}$ as

$$
\sum x_{\mu \nu} \gamma_{\nu} \mathfrak{b}_{\mu} \quad \text { with } x=\left\{x_{1}^{1}, \ldots, x_{2 k}^{1}, \ldots, x_{1}^{n}, \ldots, x_{2 k}^{n}\right\}
$$

we have

$$
\operatorname{tr}(\tau q(\mathfrak{v}))=\sum_{i} \tau^{i} q^{i}(\mathfrak{v})=x G \operatorname{diag}\left(\tau^{1} F^{1}, \ldots, \tau^{n} F^{n}\right)^{t} G^{t} x,
$$

where $\operatorname{diag}\left(\right.$ ) denotes a block diagonal matrix. We write each element $\mathfrak{l}_{\varrho}$ of the $\mathbb{Z}$ basis of $\mathfrak{L}$ as $\mathfrak{l}_{\varrho}=\sum_{\mu \nu} t_{\varrho, \mu \nu} \gamma_{\nu} \mathfrak{b}_{\mu}$, let $T=\left\{t_{\varrho, \mu \nu}\right\}$ and

$$
Z(\tau)=T G \operatorname{diag}\left(\tau^{1} F^{1}, \ldots, \tau^{n} F^{n}\right){ }^{t} G^{t} T .
$$

Then for $\mathfrak{l}$ in $\mathfrak{L}$ corresponding to row vector $l$ in $\mathbb{Z}^{d}$ we have $\operatorname{tr}(\tau q(\mathfrak{l}))=$ $Z(\tau)[l]$.

By the spectral theorem we can find matrices $S^{j}$ so that

$$
F^{j}=S^{j}\left[\begin{array}{cc}
I_{s} & 0 \\
0 & -I_{t}
\end{array}\right]{ }^{t} S^{j}
$$

Let

$$
U=T G \operatorname{diag}\left(S^{1}, \ldots, S^{n}\right)
$$


Thus $U$ is a change of basis matrix that converts $q$ into diagonal form: if $\mathfrak{l}$ in $\mathfrak{L}$ corresponds to row vector $l$ in $\mathbb{Z}^{d}$ we have

$$
\operatorname{tr}(q(\mathfrak{l}))=\operatorname{diag}\left(\ldots,\left[\begin{array}{cc}
I_{s} & 0 \\
0 & -I_{t}
\end{array}\right], \ldots\right)[l U]
$$

2. The transformation properties. We want a theta function of the form

$$
\theta(\tau, \mathfrak{r})=\sum_{\mathfrak{l} \in \mathfrak{L}+\mathfrak{r}} Q(\mathfrak{l}, v) \exp (\pi i \operatorname{tr}(\tau q(\mathfrak{l})))
$$

where $\mathfrak{r}$ is in the dual lattice $\widetilde{\mathfrak{L}}$ and $Q$, the analog of the spherical harmonic, may need to depend on the imaginary part $v$ of $\tau$. Equivalently, this can be written as

$$
\theta(\tau, \mathfrak{r})=\sum_{l \in \mathbb{Z}^{d}+r} P(l U, v) \exp (\pi i Z(\tau)[l]),
$$

where $l$ corresponds to $\mathfrak{l}$ as above, and $P$ is the function in the "diagonal coordinates" corresponding to $Q$. For such a function the equation

$$
\theta(\tau+\beta, \mathfrak{r})=\exp (\pi i \operatorname{tr}(\beta q(\mathfrak{r}))) \theta(\tau, \mathfrak{r})
$$

is obvious. The function $Q$ will have to be chosen to make the series converge absolutely and give the required inversion formula. Even in the indefinite case, the Poisson summation formula is the key to proving the inversion formula. Letting

$$
f(\tau, \mathfrak{l})=Q(\mathfrak{l}, v) \exp (\pi i \operatorname{tr}(\tau q(\mathfrak{l})))
$$

and $\sim$ denote Fourier transform, we have

$$
\begin{aligned}
\theta(\tau, \mathfrak{r}) & =\sum_{\mathfrak{l} \in \mathfrak{L}} f(\tau, \mathfrak{l}+\mathfrak{r})=\Phi^{-1 / 2} \sum_{\mathfrak{l} \in \tilde{\mathfrak{L}}}(f(\tau, \mathfrak{l}+\mathfrak{r}))^{\sim} \\
& =\Phi^{-1 / 2} \sum_{\mathfrak{l} \in \tilde{\mathfrak{L}}} \exp (\pi i\langle\mathfrak{r}, \mathfrak{l}\rangle) \tilde{f}(\tau, \mathfrak{l}) \\
& =\Phi^{-1 / 2} \sum_{\mathfrak{s} \in \tilde{\mathfrak{L}} / L} \exp (\pi i\langle\mathfrak{r}, \mathfrak{s}\rangle) \sum_{\mathfrak{l} \in \mathfrak{s}+\mathfrak{L}} \widetilde{f}(\tau, \mathfrak{l}) .
\end{aligned}
$$

We thus need to find functions $Q$ so that the corresponding $f(\tau)$ satisfies an equation like

$$
\widetilde{f}(\tau, \mathfrak{l})=i^{k n} \mathrm{n}(\tau)^{-\kappa} f(-1 / \tau, \mathfrak{l}) .
$$

Then

$$
\theta(\tau, \mathfrak{r})=\frac{i^{k n}}{\sqrt{\Phi}} \mathrm{n}(\tau)^{-\kappa} \sum_{\mathfrak{s} \in \tilde{\mathfrak{L}} / \mathfrak{L}} \exp (\pi i \operatorname{tr}(\langle\mathfrak{r}, \mathfrak{s}\rangle)) \theta(-1 / \tau, \mathfrak{s})
$$


From the above equations (2.1) and (2.3) Eichler derives the general transformation formula in Section 4 of [2].

Specializing (2.2) to $\tau=i$ we see that $f(i)$ is an eigenfunction of the Fourier transform. Thus we first look at such eigenfunctions in the next section.

3. The Hermite polynomials and parabolic cylinder functions. For completeness we will review some classical mathematics. Omitted details can be found in [3].

Hermite polynomials in one variable can be defined by

$$
H_{m}(x)=\exp \left(2 \pi x^{2}\right) D^{m}\left(\exp \left(-2 \pi x^{2}\right)\right), \quad D=\frac{d}{d x} .
$$

(This is not the standard normalization.) A generating function for the Hermite polynomials is

$$
w(x, t)=\exp \left(-2 \pi\left(2 x t+t^{2}\right)\right)=\sum_{m=0}^{\infty} \frac{H_{m}(x)}{m !} t^{m} .
$$

The identity $\partial w / \partial t+4 \pi(x+t) w=0$ gives the recurrence relation

$$
H_{m+1}(x)+4 \pi x H_{m}(x)+4 \pi m H_{m-1}(x)=0 .
$$

The identity $\partial w / \partial x+4 \pi t w=0$ and the recurrence give the differential equation

$$
H_{m}^{\prime \prime}(x)-4 \pi x H_{m}^{\prime}(x)+4 \pi m H_{m}(x)=0 .
$$

We will rewrite this equation as

$$
\left(x \frac{d}{d x}-m\right) H_{m}=\frac{1}{4 \pi} \frac{d^{2}}{d x^{2}} H_{m} .
$$

(Another linearly independent solution to the ODE can be found by applying the "reduction of order" method to the Hermite polynomial $H_{m}$. It will have exponential growth, and thus not useful for constructing theta functions. Replacing $m$ by an arbitrary parameter $\mu$ in the differential equation, we get an ODE whose solutions are the classical Hermite functions. This is not what Vignéras meant by Hermite function in [7].)

To see the significance of these polynomials, define differential operators $A=D-2 \pi x, B=D+2 \pi x$. Then the Hermite differential operator is

$$
A B-2 \pi=B A+2 \pi=D^{2}-4 \pi^{2} x^{2} .
$$

(In quantum mechanics this is the Schrödinger Hamiltonian for a harmonic oscillator.) Let $\mathcal{H}_{0}=\exp \left(-\pi x^{2}\right)$, and $\mathcal{H}_{m}=A \mathcal{H}_{m-1}$. Then

$$
\begin{aligned}
& \mathcal{H}_{m}(x)=\exp \left(\pi x^{2}\right) D^{m} \exp \left(-2 \pi x^{2}\right), \\
& \mathcal{H}_{m}(x)=\{\text { a polynomial of degree } m\} \times \exp \left(-\pi x^{2}\right), \\
& \mathcal{H}_{m}(x)=H_{m}(x) \exp \left(-\pi x^{2}\right) .
\end{aligned}
$$


The functions $\mathcal{H}_{m}(x)$ are the classical parabolic cylinder functions, up to some scaling factors. From the fact that $D$ and $-2 \pi i x$ are dual with respect to Fourier transform we get the first eigenfunction property

$$
\mathcal{H}_{m}^{\sim}(x)=(-i)^{m} \mathcal{H}_{m}(x) .
$$

The second eigenfunction property

$$
\left(D^{2}-4 \pi^{2} x^{2}\right) \mathcal{H}_{m}=-4 \pi(m+1 / 2) \mathcal{H}_{m}
$$

can be shown by induction, or proven from the connection to Hermite polynomials:

$$
H_{m}(x)=\mathcal{H}_{m}(x) \exp \left(\pi x^{2}\right)
$$

is a polynomial solution to the ODE (3.1). Use the identity

$\frac{d^{2}}{d x^{2}}\left(H(x) \exp \left(-\pi x^{2}\right)\right)=\left(-4 \pi x \frac{d H}{d x}+\frac{d^{2} H}{d x^{2}}-2 \pi H+4 \pi^{2} x^{2} H\right) \exp \left(-\pi x^{2}\right)$ to prove (3.3).

The set $\left\{\mathcal{H}_{m}\right\}$ forms (after $L^{2}$ normalization) a complete orthonormal set for $L^{2}(\mathbb{R})$. Orthogonality follows from the second eigenfunction property, since the Schrödinger Hamiltonian operator is self-adjoint. We will prove $L^{2}$ completeness in the multidimensional case below.

We want to extend the function to an arbitrary number of variables and to a quadratic form of signature $(s, t)$. Denote

- $\left(x_{1}, \ldots, x_{2 k}\right)$ the coordinates of $x \in V^{j}$ in a basis for which

$$
q(x)=\left(x_{1}^{2}+\ldots+x_{s}^{2}-x_{s+1}^{2}-\ldots-x_{2 k}^{2}\right), \quad s+t=2 k,
$$

- $s(i)= \pm 1$, the signature of $q$ at the $i$ th coordinate,

- $q_{+}(x)=\left(x_{1}^{2}+\ldots+x_{2 k}^{2}\right)$,

- $m !=m_{1} ! \ldots m_{2 k}$ !,

- $h^{\vec{m}}=h_{1}^{m_{1}} \ldots h_{2 k}^{m_{2 k}}$, and

- $\varepsilon(\vec{m})=\sum_{i=1}^{2 k} s(i) m_{i}$

where $\vec{m}=\left(m_{1}, \ldots, m_{2 k}\right) \in \mathbb{N}^{2 k}, h=\left(h_{1}, \ldots, h_{2 k}\right) \in \mathbb{R}^{2 k}$ and $\mathbb{N}$ denotes the nonnegative integers. Then the (multivariable) parabolic cylinder functions $\mathcal{H}_{\vec{m}}$ are defined by the generating function

$$
\exp \left(-2 \pi q_{+}(x+h)\right)=\exp \left(-\pi q_{+}(x)\right) \sum_{\vec{m} \in \mathbb{N}^{2 k}} \mathcal{H}_{\vec{m}}(x) \frac{h^{\vec{m}}}{m !} .
$$

This just means that $\mathcal{H}_{\vec{m}}(x)$ is a product:

$$
\mathcal{H}_{\vec{m}}(x)=\mathcal{H}_{m_{1}}\left(x_{1}\right) \ldots \mathcal{H}_{m_{2 k}}\left(x_{2 k}\right) .
$$

Since $q(x)$ is built into the inner product defining the Fourier transform, there is opportunity for confusion in looking for eigenfunctions. The multivariable Hermite function is defined as a product, so the Fourier transform 
factors as a product of Fourier transforms in each coordinate. Recall that for one variable the inverse Fourier transform is defined by $\int_{\mathbb{R}} f(y) \exp (2 \pi i x y) d y$ (up to a scalar that can be built into the measure $d y$ ), i.e. $f^{\sim \sim}(x)=f(-x)$. Thus eigenfunctions of the Fourier transform defined by the negative definite form $-x^{2}$ in one variable are the same as eigenfunctions of the classical inverse Fourier transform - the same eigenfunctions $\mathcal{H}_{n}(x)$ as above, but with the inverse eigenvalue. In the product this leads to a term $\varepsilon(\vec{m})$ :

$$
\mathcal{H}_{\vec{m}}^{\sim}=(-i)^{\varepsilon(\vec{m})} \mathcal{H}_{\vec{m}}(x) .
$$

The $\mathcal{H}_{\vec{m}}$ are solutions to a second order differential equation:

$$
\left(\Delta-4 \pi^{2} q(x)\right) \mathcal{H}_{\vec{m}}(x)=-4 \pi\left(\varepsilon(\vec{m})+\frac{s-t}{2}\right) \mathcal{H}_{\vec{m}}(x),
$$

where the Laplacian $\Delta=\sum_{i} s(i) \partial^{2} / \partial x_{i}^{2}$. The differential equation (3.5) can be justified in this multidimensional case by using the fact that $\Delta-4 \pi^{2} q(x)$ is a sum of operators acting on each coordinate, and $\mathcal{H}_{\vec{m}}(x)$ is a product.

If one considers the function $p_{\vec{m}}(x)=\mathcal{H}_{\vec{m}}(x) \exp (\pi q(x))$, then (3.5) is equivalent to

$$
(E-\varepsilon(\vec{m})+t) p_{\vec{m}}(x)=\Delta p_{\vec{m}}(x) /(4 \pi),
$$

where the Euler operator $E=\sum_{i} x_{i} \partial / \partial x_{i}$. As in equation (3.5), $E-\Delta /(4 \pi)$ is a sum of operators acting on each coordinate, and $p_{\vec{m}}(x)$ is a product. Note that at coordinates where $q(x)$ is positive, $p_{m_{i}}\left(x_{i}\right)$ is a polynomial, and we get a term $m_{i} p_{\vec{m}}(x)$ from our consideration of the one-dimensional case above. But at coordinates where $q(x)$ is negative, $p_{m_{i}}\left(x_{i}\right)$ is a polynomial times $\exp \left(-2 \pi x_{i}^{2}\right)$. Use the identity

$$
\left(4 \pi x \frac{d}{d x}+\frac{d^{2}}{d x^{2}}\right)\left(p(x) \exp \left(-2 \pi x^{2}\right)\right)=\left(\frac{d^{2} p}{d x^{2}}-4 \pi x \frac{d p}{d x}-4 \pi p\right) \exp \left(-2 \pi x^{2}\right)
$$

(where the $i$ subscript is omitted everywhere) to see the contribution to $E-\Delta /(4 \pi)$ at this coordinate is $\left(-m_{i}-1\right) p_{\vec{m}}(x)$. Summing over $i$ gives (3.6).

Proposition. The set $\left\{\mathcal{H}_{\vec{m}}\right\}$ forms a complete orthogonal basis for $L^{2}\left(\mathbb{R}^{2 k}\right)$.

Proof. Orthogonality carries over from the one-dimensional case. For completeness we follow the proof of the one-dimensional case done in exercise 16, p. 54 of [6]: If $\left\langle f, \mathcal{H}_{\vec{m}}\right\rangle=0$ for all $\vec{m}$ then

$$
\begin{aligned}
\left(f \exp \left(-\pi q_{+}()\right)\right)^{\sim}(x) & =\int f(y) \exp \left(-\pi q_{+}(y)\right) \exp (-2 \pi i\langle x, y\rangle) d y \\
& =\sum_{\vec{m} \in \mathbb{N}^{2 k}}(-1)^{\delta(\vec{m})} \frac{(2 \pi i x)^{\vec{m}}}{m !} \int y^{\vec{m}} \exp \left(-\pi q_{+}(y)\right) f(y) d y .
\end{aligned}
$$

(Here $\delta(\vec{m})=\sum_{s(i)<0} m_{i}$.) Now $y^{\vec{m}} \exp \left(-\pi q_{+}(y)\right)$ is a linear combination of 
the functions $\mathcal{H}_{\vec{n}}(y)$ such that $\sum n_{i} \leq \sum m_{i}$. This is clear from the recursion in the one-dimensional case and follows in general by the multiplicativity. By the orthogonality hypothesis

$$
\left(f \exp \left(-\pi q_{+}()\right)\right)^{\sim}(x)=0,
$$

and thus $f=0$ as Fourier transform is injective in the $L^{2}$ sense. (The paper of Appell and Kampé de Fériet [1] which is cited in [7] shows only that Hermite polynomials of degree less than $n$ provide the best polynomial approximation in the relevant inner product. This leads to Bessel's inequality, but does not prove completeness.)

For $\tau=u+i v$ in the complex upper half plane $\mathbf{H}$, let

$$
\mathcal{H}_{\vec{m}}(\tau, x)=v^{(t-\varepsilon(\vec{m})) / 2} \mathcal{H}_{\vec{m}}(x \sqrt{v}) \exp (\pi i q(x) u) .
$$

LEMMA.

$$
\mathcal{H}_{0}^{\sim}(\tau, x)=(-i)^{(t-s) / 2} \tau^{(t-s) / 2} \mathcal{H}_{0}(-1 / \tau, x) .
$$

(Note that $s+t=2 k$, so $t-s \equiv 0 \bmod 2$.)

Proof. It is well known that

$$
\int_{\mathbb{R}} \exp \left(\pi i y^{2} \tau\right) \exp (-2 \pi i x y) d y=(\tau / i)^{-1 / 2} \exp \left(-\pi i x^{2} / \tau\right) .
$$

To generalize to several variables, let

$$
\begin{aligned}
g(\tau, x)=\exp \left(\pi i q(x) u-\pi q_{+}\right. & (x) v) \\
& =\prod_{s(i)>0} \exp \left(\pi i x_{i}^{2} \tau\right) \prod_{s(i)<0} \exp \left(\pi i x_{i}^{2}(-\bar{\tau})\right) .
\end{aligned}
$$

Then

$$
\begin{aligned}
g^{\sim}(\tau, x) & =(\tau / i)^{-s / 2}(-\bar{\tau} / i)^{-t / 2} g(-1 / \tau, x) \\
& =(-i)^{(t-s) / 2} \tau^{(t-s) / 2}\left(u^{2}+v^{2}\right)^{-t / 2} g(-1 / \tau, x) .
\end{aligned}
$$

By considering $v^{t / 2} g(\tau, x)$, we eliminate the $\left(u^{2}+v^{2}\right)^{-t / 2}$ term:

$$
v^{t / 2} g^{\sim}(\tau, x)=(-i)^{(t-s) / 2} \tau^{(t-s) / 2}(\operatorname{Im}(-1 / \tau))^{t / 2} g(-1 / \tau, x) .
$$

Equivalently, the Fourier transform of the function $\mathcal{H}_{0}(\tau, x)=v^{t / 2} g(\tau, x)$ is

$$
\mathcal{H}_{0}^{\sim}(\tau, x)=(-i)^{(t-s) / 2} \tau^{(t-s) / 2} \mathcal{H}_{0}(-1 / \tau, x) .
$$

We generalize this to arbitrary $\vec{m}$ :

Lemma. With $\lambda=\varepsilon(\vec{m})-t$,

$$
\mathcal{H}_{\vec{m}}^{\sim}(\tau, x)=(-i)^{(t-s) / 2} \tau^{-(\lambda+k)} \mathcal{H}_{\vec{m}}(-1 / \tau, x) .
$$

Proof. The identity

$$
\frac{\partial}{\partial x_{i}} \mathcal{H}_{\vec{m}}(x)-2 \pi x_{i} \mathcal{H}_{\vec{m}}(x)=\mathcal{H}_{\vec{m}+1}(x)
$$


(where $\vec{m}+1$ really means add 1 to $\vec{m}$ in the $i$ th coordinate) defined the one variable case and carries over by multiplicitivity. Now compute $\frac{\partial}{\partial x_{i}} \mathcal{H}_{\vec{m}}(\tau, x)$ and combine with the above identity to see that

$$
v \mathcal{H}_{\vec{m}+1}(\tau, x)=\frac{\partial}{\partial x_{i}} \mathcal{H}_{\vec{m}}(\tau, x)-2 \pi i x_{i} \bar{\tau} \mathcal{H}_{\vec{m}}(\tau, x) .
$$

Since

$$
\left(\frac{\partial}{\partial x_{i}} f\right)^{\sim}=s(i) 2 \pi i x_{i} f^{\sim} \quad \text { and } \quad\left(2 \pi i x_{i} f\right)^{\sim}=-s(i) \frac{\partial}{\partial x_{i}} f^{\sim},
$$

induction gives the lemma.

4. More on the transformation properties. We can now answer the question posed at the end of Section 2. Choose any $\vec{m}^{j} \in \mathbb{N}^{2 k}, j=1, \ldots, n$. Then the product

$$
f(\tau, x)=\prod_{j} \mathcal{H}_{\vec{m}^{j}}\left(\tau^{j}, x^{j}\right)
$$

with $x=\left\{x^{1}, \ldots, x^{n}\right\}=\left\{x_{1}^{1}, \ldots, x_{2 k}^{1}, \ldots, x_{1}^{n}, \ldots, x_{2 k}^{n}\right\}$ satisfies an equation similar to $(2.2)$ :

$$
\widetilde{f}(\tau, x)=(-i)^{n(t-s) / 2} \mathrm{n}(\tau)^{-(\lambda+k)} f(-1 / \tau, x),
$$

where $\mathrm{n}(\tau)^{-(\lambda+k)}$ is sloppy notation for $\prod_{j}\left(\tau^{j}\right)^{-\left(\varepsilon\left(\vec{m}^{j}\right)-t+k\right)}$. So we can build a theta function with it and get the transformation law we want. More generally, we get the following

THEOREM 1. Suppose the functions $p_{j}$ satisfy the differential equation

$$
\left(E-\lambda_{j}\right) p_{j}(x)=\Delta p_{j}(x) /(4 \pi),
$$

and also

$$
p_{j}\left(x^{j}\right) \exp \left(-\pi q\left(x^{j}\right)\right) \in L^{2}\left(\mathbb{R}^{2 k}\right)
$$

for $j=1, \ldots, n$. For $\mathfrak{l} \in \mathfrak{L}$ corresponding to $l=\left(\ldots, l^{j}, \ldots\right) \in \mathbb{Z}^{d}$ let

$$
Q(\mathfrak{l}, v)=\prod_{j}\left(v^{j}\right)^{-\lambda_{j} / 2} p_{j}\left((l U)^{j} \sqrt{v^{j}}\right),
$$

where $U$ is the diagonalizing operator of Section 1 . Then

$$
\theta(\tau, \mathfrak{r})=\sum_{\mathfrak{l} \in \mathfrak{L}+\mathfrak{r}} Q(\mathfrak{l}, v) \exp (\pi i \operatorname{tr}(\tau q(\mathfrak{l})))
$$

is an automorphic form of weight $\left(\ldots, \lambda_{j}+k, \ldots\right)$. The level and character are as in Eichler [2]. (See also Walling [8] for details on the character at dyadic primes.)

Summary of proof. We showed that for $p_{j}$ to satisfy (3.6) is equivalent to $p_{j}\left(x^{j}\right)$ times $\exp \left(-\pi q\left(x^{j}\right)\right)$ satisfying the corresponding version of 
(3.5). Given also the $L^{2}$ condition (4.1), $p_{j}\left(x^{j}\right) \exp \left(-\pi q\left(x^{j}\right)\right)$ can be written as an (infinite) combination of the functions $\mathcal{H}_{\vec{m}}\left(x^{j}\right)$ with $\lambda_{j}=\varepsilon(\vec{m})+t$. Then the corresponding function

$$
\left(v^{j}\right)^{-\lambda_{j} / 2} p_{j}\left(x^{j} \sqrt{v^{j}}\right) \exp \left(\pi i q\left(x^{j}\right) \tau^{j}\right)
$$

will be the same linear combination of the functions $\mathcal{H}_{\vec{m}}\left(\tau^{j}, x^{j}\right)$. Taking a product over $j=1, \ldots, n$ we see that the function

$$
f(\tau, \mathfrak{l})=Q(\mathfrak{l}, v) \exp (\pi i \operatorname{tr}(\tau q(\mathfrak{l})))
$$

satisfies (2.2):

$$
\widetilde{f}(\tau, \mathfrak{l})=(-i)^{n(t-s) / 2} \mathrm{n}(\tau)^{-(\lambda+k)} f(-1 / \tau, \mathfrak{l})
$$

because it is a sum of terms $\prod_{j} \mathcal{H}_{\vec{m}^{j}}\left(\tau^{j}, x^{j}\right)$ which do. Eichler has shown the automorphic property follows once (2.1) and (2.3) are proven.

5. An application: relative quadratic base change. Let $F$ be a totally real quadratic extension of $K$ with Galois automorphism $\sigma$. Let $i$ denote the involution $\left[\begin{array}{ll}a & b \\ c & d\end{array}\right] \rightarrow\left[\begin{array}{cc}d & -b \\ -c & a\end{array}\right]$, and consider the vector space

$$
V=\left\{x \in M_{2}(F) \mid x^{i}=-{ }^{\sigma} x\right\} .
$$

So $x \in V$ looks like $\left[\begin{array}{cc}x_{1} & x_{4} \\ x_{3}-\sigma_{x_{1}}\end{array}\right]$ with $x_{3}, x_{4} \in K$ and $x_{1} \in F$. We take the quadratic form of signature $(2,2)$ defined by

$$
q(x)=-2 \operatorname{det}(x) \quad \text { on } \mathfrak{L}=V \cap M_{2}\left(\mathcal{O}_{F}\right) .
$$

The corresponding bilinear form is $\langle x, y\rangle=-\operatorname{tr}\left(x y^{i}\right)$. Then for $z_{1}, z_{2} \in \mathcal{H}^{n}$, $z^{j}=\left[\begin{array}{cc}-z_{2} & z_{1} z_{2} \\ -1 & z_{1}\end{array}\right]^{j}$ satisfies $q\left(z^{j}\right)=0$ and $\left\langle z^{j}, \bar{z}^{j}\right\rangle>0$. And

$$
\left\langle x^{j}, z^{j}\right\rangle=\left(x_{3} z_{1} z_{2}-x_{1} z_{1}+x_{2} z_{2}-x_{4}\right)^{j} \quad \text { for } x=\left[\begin{array}{ll}
x_{1} & x_{4} \\
x_{3} & x_{2}
\end{array}\right] .
$$

With respect to the standard basis of $M_{2}(\mathbb{R})$, the Laplacian associated with $q(x)$ is $\Delta=\partial^{2} / \partial x_{3} \partial x_{4}-\partial^{2} / \partial x_{1} \partial x_{2}$. One can verify that for $k \geq 4$,

$$
p_{j}(x)=q^{j}(x)^{k-1}\left\langle x^{j}, z^{j}\right\rangle^{-k}
$$

satisfies $\Delta p_{j}=0$ and $E p_{j}=(k-2) p_{j}$. (Here the dimension of the vector space is 4 , and now $k$ will be the weight of the automorphic form.)

Thus (3.6) of Theorem 1 applies with each $\lambda_{j}=k-2$. However, since $q(x)$ is indefinite there is no chance that property (4.1) will hold. Instead we consider the function

$$
p_{j}(x)= \begin{cases}\frac{q^{j}(x)^{k-1}}{\left\langle x^{j}, z^{j}\right\rangle^{k}} & \text { if } q^{j}(x)>0 \\ 0 & \text { if } q^{j}(x) \leq 0\end{cases}
$$


One can check that for $k \geq 4$, all partial derivatives of order two or less of $q(x)^{k-1}\langle x, z\rangle^{-k}$ are 0 on the conic $q(x)=0$; so $p_{j}(x)$ also satisfies (3.6) and (4.1) holds trivially. And $p_{j}(x)$ is a homogeneous function by the following

Well Known Lemma. For "nice" functions $p: \mathbb{R}^{n} \rightarrow \mathbb{C}, p(c x)=$ $c^{\lambda} p(x)$ for all real $c$ if and only if $E p=\lambda p$.

Proof. First take derivatives with respect to $c$ in $p(c x)=c^{\lambda} p(x)$, then multiply by $c$ to get

$$
\sum_{i} c x_{i} p_{x_{i}}(c x)=\lambda c^{\lambda} p(x)=\lambda p(c x) .
$$

This hold true for all $c$ and $x$; now take $c=1$. Conversely,

$$
\frac{d}{d c} p(c x)=\sum_{i} x_{i} p_{x_{i}}(c x)=\frac{\lambda}{c} p(c x) \quad \text { by Euler, }
$$

or

$$
\frac{\frac{d}{d c} p(c x)}{p(c x)}=\frac{\lambda}{c} .
$$

Thus $\log (p(c x))=\lambda \log (c)+\kappa$ (up to multiples of $2 \pi i$ ). So $p(c x)=c^{\lambda} \exp (\kappa)$ and $c=1$ shows that $\exp (\kappa)=p(x)$.

Homogeneity is very powerful; it means the function $Q$ no longer depends on the imaginary part $v$ of $\tau$, and thus the theta function will be holomorphic. In particular, we get

THEOREM 2.

$$
\begin{aligned}
\Omega\left(\tau, z_{1}, z_{2}\right) & =\sum_{\substack{x \in \mathfrak{L} \\
q(x) \gg 0}} \mathrm{n}\left(q(x)^{k-1}\langle x, z\rangle^{-k}\right) \exp (\pi i \operatorname{tr}(q(x) \tau)) \\
& =\sum_{\substack{x \in \mathfrak{L} \\
q(x) \gg 0}} \mathrm{n}\left(\frac{\left(x_{3} x_{4}+x_{1}{ }^{\sigma} x_{1}\right)^{k-1}}{\left(x_{3} z_{1} z_{2}-x_{1} z_{1}-{ }^{\sigma} x_{1} z_{2}-x_{4}\right)^{k}}\right) \exp (\pi i \operatorname{tr}(q(x) \tau))
\end{aligned}
$$

is a holomorphic Hilbert modular form of constant weight $k$ with level $\mathcal{N}$ and character $\chi$ as in Eichler [2].

The lifting is now obtained as usual by integrating cusp forms of level $\mathcal{N}$ and character $\chi$ against the kernel $\Omega$. In particular, integrating against the $\nu$ th Poincaré series gives

$$
\mathrm{n}(2 \nu)^{k-1} \sum_{\substack{x \in \mathfrak{L} \\ q(x)=2 \nu}} \mathrm{n}\left(\langle x, z\rangle^{-k}\right) .
$$


This sum converges absolutely; indeed, for any $p$ satisfying (4.1), we have

$$
\begin{aligned}
\sum_{\substack{x \in \mathfrak{L} \\
q(x)=2 \nu}}|p(x)| & =|\exp (\pi 2 \nu)| \sum_{\substack{x \in \mathfrak{L} \\
q(x)=2 \nu}}|p(x) \exp (-\pi q(x))| \\
& \ll|\exp (\pi 2 \nu)| \sum_{x \in \mathfrak{L}}|p(x) \exp (-\pi q(x))| \ll \infty .
\end{aligned}
$$

No term of the product $\mathrm{n}\left(\langle x, z\rangle^{-k}\right)$ vanishes for $\left(z_{1}, z_{2}\right) \in \mathcal{H}^{n} \times \mathcal{H}^{n}$ (as $x_{3} z_{1} z_{2}-x_{1} z_{1}-{ }^{\sigma} x_{1} z_{2}-x_{4}=0$ implies $z_{2}=\left(x_{1} z_{1}+x_{4}\right) /\left(x_{3} z_{1}-{ }^{\sigma} x_{1}\right)$ but $\operatorname{det}(x)=-2 \nu \ll 0$ and $\left.z_{1}, z_{2} \in \mathbf{H}\right)$; thus the series defines a holomorphic function by the absolute convergence. The lifted Poincare series is a form of weight $k$ for $S L\left(2, \mathcal{O}_{F}\right)$; the arguments in [9] carry over.

\section{References}

[1] P. Appell et J. Kampé de Fériet, Fonctions Hypergéométriques et Hypersphériques (troisième partie: Polynomes d'Hermite...), Gauthier-Villars, Paris, 1926.

[2] M. Eichler, On theta functions of real algebraic number fields, Acta Arith. 33 (1977), 269-292.

[3] N. Lebedev, Special Functions and their Applications, Dover, 1972.

[4] W. Schemp p, Harmonic Analysis on the Heisenberg Nilpotent Lie Group, Longman, 1986.

[5] C. L. Siegel, Indefinite quadratische Formen und Funktionentheorie I, Math. Ann. 124 (1951), 17-54; II , ibid., 364-387.

[6] A. Terras, Harmonic Analysis on Symmetric Spaces and Applications I, Springer, 1985.

[7] M.-F. Vignéras, Séries thêta des formes quadratiques indéfinies, in: Modular Functions of One Variable VI, Lecture Notes in Math. 627, Springer, 1977, 227-239.

[8] L. H. Walling, Hecke operators on theta series attached to lattices of arbitrary rank, Acta Arith. 54 (1990), 213-240.

[9] D. Zagier, Modular forms associated to real quadratic fields, Invent. Math. 30 (1975), $1-46$.

MATHEMATICS DEPARTMENT

UNIVERSITY OF CALIFORNIA

SANTA BARBARA, CALIFORNIA 93106

U.S.A.

E-mail: STOPPLE@MATH.UCSB.EDU 